\title{
Phase-measuring time-gated BOCDA
}

\author{
Alexia López-Gil, ${ }^{1, *}$ Sonia Martin-Lopez, ${ }^{1}$ and Miguel GonZalez-HerRaez ${ }^{1}$ \\ ${ }^{1}$ Dept. of Electronics, University of Alcala, Polytechnic School, 28805 Alcala de Henares, Spain \\ *Corresponding author: alexia.lopez@uah.es
}

Received XX Month XXXX; revised XX Month, XXXX; accepted XX Month XXXX; posted XX Month XXXX (Doc. ID XXXXX); published XX Month XXXX

\begin{abstract}
We demonstrate a simple scheme allowing to perform distributed Brillouin Phase Shift (BPS) measurements with very high spatial resolution $(\sim 7 \mathrm{~cm})$ over long $(\sim 4.7$ km) optical fibers. This is achieved by inserting a Sagnac interferometer (SI) in a Brillouin Optical Correlation Domain Analysis (BOCDA) configuration. Over its already-presented time-domain equivalent (SI-BOTDA), this approach reduces the main source of noise (coherent backscatter noise) thanks to the low-coherence nature of the used signals. On the other hand, over the most usual schemes used for distributed BPS measurements, this implementation presents the key advantage of not requiring high-bandwidth detection or complex modulation, while reaching unprecedented values of spatial resolution and number of resolved points for this type of measurements. Thanks to the linear dependence of the BPS feature around the Brillouin Frequency Shift (BFS), this scheme could also have the advantage of requiring shorter scanning ranges than amplitude-based configurations.
\end{abstract}

\section{(c) 2017 Optical Society of America}

OCIS codes: (060.2370) Fiber optics sensors; (290.5900) Scattering, stimulated Brillouin; (060.4370) Nonlinear optics, fibers; (280.6780) Temperature, (120.3180) Interferometry.

http://dx.doi.org/10.1364/OL.99.099999

Stimulated Brillouin scattering (SBS) in optical fibers offers a means for distributed temperature and strain monitoring [1]. When a coherent light beam is introduced on one end of the optical fiber, SBS manifests as two anti-symmetric counter-propagating frequency-shifted narrowband amplification and attenuation curves. The frequency offset of the two resonances from the input beam frequency is called the Brillouin Frequency Shift (BFS). The linear dependence with strain and temperature of the BFS has allowed developing Brillouin distributed fiber sensors. The Brillouin gain/loss spectra (BGS) entail an associated phase response called the Brillouin phase spectrum (BPS) [1]. Together with the BGS, the associated BPS varies as well its position with any inhomogeneity in the fiber, which makes it also suitable for sensing purposes [2]. Moreover, recently it has been demonstrated that, over homogeneous fibers, the spectral range required to achieve the same BFS determination error is smaller when using BPS instead of BGS [3].

We recently proposed a simple, baseband and filter-less measurement scheme (SI-BOTDA) to provide the distributed BPS feature in Brillouin optical time domain analysis (BOTDA) systems. The technique was based on using a Sagnac interferometer (SI) as a homodyne interferometer in a standard BOTDA structure $[4,5]$ The use of a dual sideband in this case allowed a convenient measurement of the BPS response using intensity-only measurements. Unfortunately, including a laser-driven SI within the BOTDA structure also increases very substantially the Rayleigh coherent backscatter noise [6] of the system. In this Letter, we demonstrate a simple scheme to perform BPS measurements with very sharp spatial resolution $(\sim 7 \mathrm{~cm})$ over long $(\sim 4.7 \mathrm{~km})$ optical fibers by inserting a SI in a Brillouin Optical Correlation Domain Analysis (BOCDA) configuration. To the best of our knowledge, this is the first demonstration of a time-gated BOCDA based on BPS measurements. Over its corresponding time-domain scheme (SIBOTDA), this approach reduces several orders of magnitude the correlation noise thanks to the low-coherence nature of the used signals [6], while achieving resolution values that are two orders of magnitude better ( $\mathrm{cm}$-scale resolution and $>67000$ resolved points in this implementation). Moreover, the SI-BOCDA implementation presents the key advantage of not requiring highbandwidth detection or complex modulation [2, 7-10], which are typical in distributed BPS measurements. In addition, thanks to using the BPS instead of the BGS, this scheme could also have the advantage of requiring shorter scanning ranges (particularly in uniform fibers) than BGS-based configurations [3].

As mentioned above, our proposal is based on a BOCDA configuration, specifically on a time-gated BOCDA scheme [11, 12]. The core of a high-resolution time-gated BOCDA is the correlated modulation of the interacting optical waves, pump and probe, which confines the Brillouin interaction to the correlation peaks, in a similar way to a conventional BOCDA. However, in the timegated configuration the amplitude of the pump wave is also modulated by a single pulse to allow the location of multiple correlation peaks along the fiber, instead of only one. The length of the pump pulse cannot be longer than the separation between correlation peaks to allow for the isolation of a single peak at each position. Therefore, multiple correlation peaks can be simultaneously interrogated by performing a time-resolved measurement. Two different implementations have been typically 
used in these schemes: one in which the pump and the probe signals are jointly modulated by a sine wave [11] and another one in which a coded phase sequence is used [12].

Theoretically, considering the implementation in which a coded phase sequence is employed, the spatial resolution of the correlation peaks is on the order of $\Delta z=v_{g} T / 2$, where $v_{g}$ is the group velocity of light in the fiber and $T$ is the symbol duration (typically much shorter than the phonon lifetime), and the interval between adjacent correlation peaks equals $v_{g} /\left(2 f_{m}\right)=\left(M v_{g} T\right) / 2$, where $f_{m}$ is the modulation frequency ( $f_{m}=1 / M T, M$ being the number of symbols of the sequence). Unlike the implementation in which a sine wave is employed, the unambiguous measurement range can thus be made arbitrarily long, while retaining a sharp spatial resolution $\Delta z$. In our approach, we use a direct, low-depth modulation of the master laser using a coded sequence (i.e. without employing an external phase modulator as it is usually done [12]). This entails one major advantage over the coded phase approach (we avoid using a phase modulator and its associated driver) and one major drawback (the bandwidth of the modulating signal is not fully exploited, and the resolution does not correspond to the nominal resolution of the bit period). The position of each peak, except for the central one, is swept by changing the modulation frequency (or equivalently, $T$ ) in order to obtain a measurement of the whole fiber. It should be taken into account that the position shift caused by the change of modulation frequency is proportional to the order of each peak. To reduce the step irregularity, a long delay fiber is placed in one of the arms of the scheme, so that the orders of the peaks within the FUT are high. Finally, the Brillouin spectrum at each correlation peak could be obtained by sweeping the pump-probe frequency shift around the BFS of the fiber.

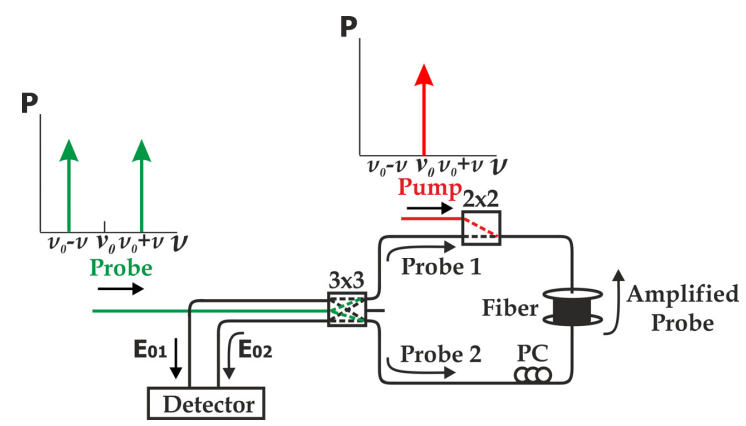

Fig. 1: Scheme of a Brillouin distributed sensor with a Sagnac interferometer within the tested fiber. E01: Output electric field 1; E02: Output electric field 2; PC: Polarization Controller. $v_{0}$ : pump frequency.

To obtain the BPS distribution along the fiber, we use a SI as a homodyne interferometer in the time-gated BOCDA setup previously described $[4,5]$. A dual sideband probe wave is introduced in the SI using a $3 \times 3$ coupler. The two counterpropagating probe waves (Probe 1 and 2) travel in opposite directions within the fiber, thus interfering at the output of the remaining ports of the SI (Fig. 1). A certain non-reciprocal phase shift will arise between the two counter-propagating paths followed by the probe light in the SI. The SBS pump is made to copropagate with Probe 1 . Thus, only Probe 2 will experience the SBS process; the lower frequency band will be amplified while the upper one attenuated, both of them suffering the same nonreciprocal BPS. For the typical case of small gain, the outputs of the SI will depend only on the shape of the phase shift and not on the gain or attenuation (see the model developed in [5]). Our developed experimental setup (Fig. 2) is similar to the SI-BOTDA setup used in [5] except for the fact that a direct current modulation of the master laser is carried out, in this case driven by a PRBS (pseudo random bit sequence) generator. This modulation scheme relies on the non-ideal behavior (chirp) of semiconductor lasers with the applied current. In this case, the applied current modulation is extremely small in comparison with the bias current, leading to an almost phase-coded system with no major amplitude noise. This method is cheaper than conventional phase coding (as no external modulator or driver is required), however it provides a deteriorated resolution over the conventional method, related to the bandwidth limitations of direct current modulation of semiconductor lasers. As in most Brillouin sensor schemes, light from a single Distributed Feedback (DFB) laser diode at $1550 \mathrm{~nm}$ is used as a common source for both pump and probe waves. The generator clock rate $(1 / T)$ is in the range of $10.024 \mathrm{GHz}$, and the code length is $M=2^{15}-1$. The symbol duration is slightly swept with a step of $10 \mathrm{KHz}$ to move simultaneously 15 correlation peaks along the whole fiber length. The distance between correlation peaks is $\sim 327 \mathrm{~m}$. The frequency-modulated light is split into pump and probe branches. The probe wave is obtained through an intensity modulation of the master laser yielding a double sideband (DSB) probe spectrum with no carrier. The modulating frequency is chosen to sweep around the BFS of the FUT. $~ 1.9$ $\mathrm{mW}$ probe sidebands are launched into the SI through the $3 \times 3$ coupler. As the position of the correlation peaks depends on the peak order, in the pump arm a $\sim 50 \mathrm{~km}$ delay fiber is inserted to reduce the step irregularity. This means that relatively high-order correlation peaks (68th-83rd) are located in the optical fiber. Note that this delay line has no impact in terms of nonlinearities, as the pump power crossing the delay fiber is fairly small (in the sub-mW range). The pump light is amplitude-modulated into sharp square pulses of $70 \mathrm{~ns}$ (100 ps fall time) using a semiconductor optical amplifier (SOA) connected to a pulse generator. The pulses are then amplified by an EDFA. The pulse repetition period is $50 \mu \mathrm{s}$. An electro-optic microelectromechanical system (MEMS) switch is used to gate the pulses and ensure an overall extinction ratio (ER) higher than $80 \mathrm{~dB}$. A band-pass optical filter (BPOF) is used to eliminate most of the amplified spontaneous emission (ASE) noise introduced by the EDFA. Upon entrance into the SI through the coupler, the pulses have a peak power of $\sim 60 \mathrm{~mW}$. The pulses are polarization-scrambled to reduce the polarization sensitivity of the SBS interaction. Another SOA acting as a switch is placed within the SI, gated to block the residual pump power, appearing in the middle of the FUT temporal trace [5]. An AC signal proportional to the SBS phase shift can be extracted from either light channel at the output of the SI. These fields are then introduced into the positive and negative ports of a balanced detection system (100 MHz bandwidth) obtaining an increased amplitude in the Brillouin phase signal, while being robust to common-mode intensity noise [13]. The sampling interval of the oscilloscope is set to $4 \mathrm{~cm}(400$ ps).

In order to analyze the performance of the above explained technique, interferometric time-gated BOCDA measurements have been carried out over a $4.7 \mathrm{~km}$ single-mode fiber (composed of 


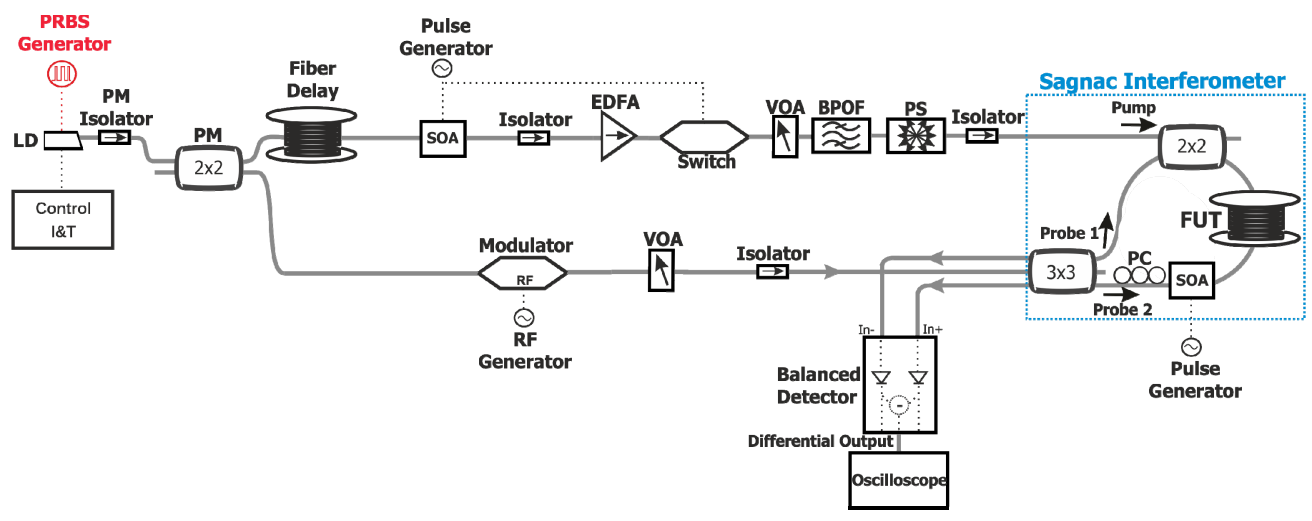

Fig. 2: Schematic drawing of the interferometric time-gated BOCDA. PRBS: Pseudo Random Bit Sequence; LD: Laser Diode; PM: Polarization Maintaining; SOA: Semiconductor Optical Amplifier; EDFA: Erbium Doped Fiber Amplifier; VOA: Variable Optical Attenuator; BPOF: Band-Pass $\begin{array}{llllllllll}\text { optical Filter; } & \text { PS: } & \text { Polarization } & \text { Scrambler; } & \text { PC: } & \text { Polarization } & \text { Controller; } & \text { FUT: } & \text { Fiber } & \text { Under }\end{array}$

three spools of $4.2 \mathrm{~km}, 300$ and $200 \mathrm{~m})$. The BFS of the spool of 4.2 $\mathrm{km}$ is $\sim 10.88 \mathrm{GHz}$ and the BFS of the other two spools is $\sim 10.858$ $\mathrm{GHz}$ (at $1550 \mathrm{~nm}$ ).

In Fig. 3, two examples of $\sim 1100 \mathrm{~m}$ of the temporal trace of the first fiber obtained at the output of the SI for a pump-probe frequency of $10.875 \mathrm{GHz}$ (a) and $10.895 \mathrm{GHz}$ (b) are depicted. It can be seen that, when the pump-probe frequency shift is lower than the average BFS of the optical fiber, the correlation peaks point downwards, while, when the pump-probe frequency shift is higher than the BFS, the correlation peaks point upwards. As mentioned, the signal at each correlation peak is proportional to the induced phase shift at that position, which can be positive or negative depending on the relative position of the probe sidebands with respect to the SBS resonance.

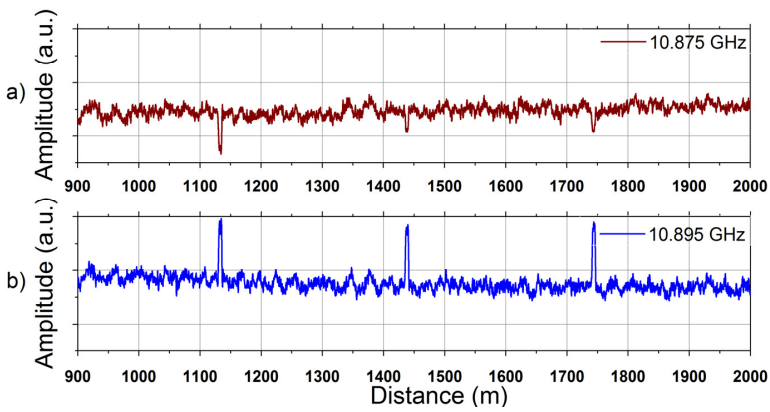

Fig. 3: Detail of $\sim 1100 \mathrm{~m}$ (first optical fiber) of the temporal trace obtained for a pump-probe frequency shift of: a) $10.875 \mathrm{GHz}$ and b) $10.895 \mathrm{GHz}$

The performance of the setup as a temperature sensor has been finally evaluated by creating a $25 \mathrm{~cm}$ hot-spot at the end of the FUT (around $4.5 \mathrm{~km}$ ) with a heating resistor attached. A high enough current is applied to heat the fiber up to $\sim 70{ }^{\circ} \mathrm{C}$. The probe wave is swept from $10.80 \mathrm{GHz}$ until $10.95 \mathrm{GHz}$ with a $1 \mathrm{MHz}$ step, where each temporal trace is averaged 4096 times. Fig. 4 shows the results of temperature change around the hot-spot (red) in comparison with the same location in a room with a temperature of $28{ }^{\circ} \mathrm{C}$ (green). The heated section shows a BFS at $\sim 10.90 \mathrm{GHz}$ offseted by $\sim 42 \mathrm{MHz}$ when compared to the rest of the fiber at room temperature. Considering a sensitivity of $1 \mathrm{MHz} /{ }^{\circ} \mathrm{C}$ in the BFS, this gives us a temperature variation of $\sim 42{ }^{\circ} \mathrm{C}$, which is in good agreement with the expected temperature difference.

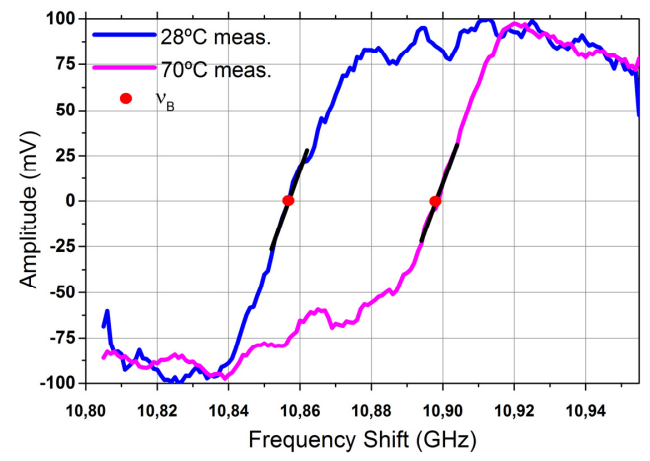

Fig. 4: Experimental results of the phase profiles for a fiber region with normal temperature $\left(28^{\circ} \mathrm{C}\right)$ and a heated region around $\sim 25 \mathrm{~cm}$ hotspot (located around $4.5 \mathrm{~km}$ ).

To confirm the possibility of using this scheme to perform distributed BPS measurements, a full 3D map of the retrieved phase shape for the complete fiber length has been obtained. In Fig. 5, we depict a detail of $45 \mathrm{~cm}$ of this 3D map around the heated region (at $4.5 \mathrm{~km}$ ). As it can be inferred from this figure, the hotspot is correctly identified as being $\sim 25 \mathrm{~cm}$ wide. The hot-spot has a transient distance of $\sim 7 \mathrm{~cm}$, pointing to the achievable spatial resolution of our sensor, which was confirmed by standard (gain/loss) BOCDA measurements. Note that the spatial resolution of our scheme is not as high as the one that could be obtained with a phase-correlation distributed Brillouin sensor using external modulation [12] under the same conditions (1 cm using 10 Gbps modulation). As mentioned, this limitation is related to the bandwidth limits of directly modulated semiconductor lasers. Nevertheless, the SI technique can still be successfully applied to any BOCDA sensor, not only to those directly modulated in frequency with a PRBS generator.

The evolution of the BFS along the optical fiber, from which the variations of temperature can be extracted, is usually obtained by 
performing a linear fit around the zero de-phase frequency region of the BPS locally measured at each fiber position [3]. In our setup, the number of scanned spectral points around the BFS employed to perform this fitting is 11 that corresponds to the use of a quarter of the full-width at half-maximum (FWHM) of the BGS with a sampling step of $1 \mathrm{MHz}$. The frequency uncertainty measured around the hot-spot is $\sim 3.01 \mathrm{MHz}$. Under these conditions the quadratic fitting over the BGS would provide a significantly higher BFS determination error (approximately two times the error using the BPS profile) as demonstrated in [3]. This may reduce the measurement time of specific high resolution BOCDA systems requiring a narrow frequency scanning (homogeneous fiber with small BFS variations).

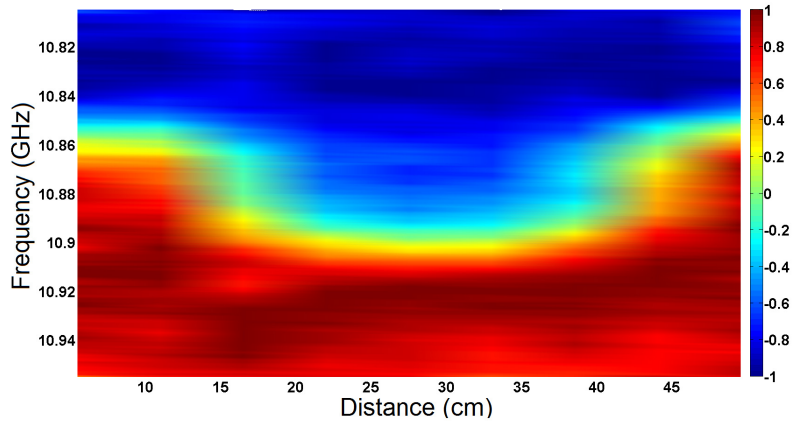

Fig. 5: BPS sweep around $4.5 \mathrm{~km}$ of the fiber showing a $25 \mathrm{~cm}$ hot-spot presenting a BFS offset by $\sim 42 \mathrm{MHz}$ with respect to the unheated fiber. The probe signal frequency is swept from $10.80 \mathrm{GHz}$ to $10.95 \mathrm{GHz}$, and the traces are acquired with $70 \mathrm{~ns}$ pump pulses. The number of averages is 4096.

It should be also highlighted that just utilizing a proper filtering process before the detector and linear combinations of the retrieved signals, the sensor could measure simultaneously the distributed BGS and BPS features which could eventually provide redundancy and increase the resilience of the system to BFS determination errors [14].

Lastly, it is interesting to compare this setup with its timedomain counterpart (SI-BOTDA) [5]. The SI-BOTDA had a significant shortcoming related to the presence of additional coherent Rayleigh noise in comparison with the standard BOTDA scheme. Interestingly, this noise, which is dominant in SI-BOTDA, can be substantially lowered by reducing the coherence length of the light source driving the system [6] (for example using a PRBS modulation of the input laser). To quantify this Rayleigh noise reduction, it is interesting to compare the normalized SNR in both cases (measuring the noise as the standard deviation of the difference among an ideal theoretical normalized phase spectrum and the measured one at the same position and under the same conditions [3]). The standard deviation computed over a $30-\mathrm{MHz}$ window in this case is $\sim 0.038$, while it is $\sim 0.077$ in the SI-BOTDA scheme with a resolution of $2.5 \mathrm{~m}$ [5]. Consequently, it must be noted that the presented setup, despite having a spatial resolution approximately $\sim 35$ times lower $(\sim 7 \mathrm{~cm})$ than the SIBOTDA system $(\sim 2.5 \mathrm{~m})$, presents a normalized SNR similar to the other scheme. Actually the correlation scheme presents a twofold improvement in SNR simply due to the fact that the noise is decreased proportionally to $\sqrt{N_{a v g}}$, and $N_{\text {avg }}$ (the number of averages performed on the time traces) is 4 times greater in the present setup (4096) than in the SI-BOTDA scheme (1024).

In summary, the present contribution demonstrates the proof of concept of a time-gated BOCDA capable of delivering BPS measurements by just introducing a SI in the scheme. The presented technique is, to the best of our knowledge, the first demonstration of a phase-measuring time-gated BOCDA. The fundamentals of the technique have been described and validated through experimental measurements. In contrast to the most usual schemes used for distributed BPS measurements, this setup presents the key advantage of not requiring high-bandwidth detection, complex modulation or sharp filtering, while reaching unprecedented values of spatial resolution $(\sim 7 \mathrm{~cm})$ and number of resolved points $(\sim 67143)$ for distributed BPS measurements. In addition, in terms of coherent Rayleigh backscatter noise, the present method yields an important improvement when compared to our previous time-domain BPS measuring approach [5] owing to the low-coherence nature of the used signals.

Funding. H2020 European Research Council (ERC) (307441); FINESSE (MSCA-ITN-ETN-722509); DOMINO Water JPI project; Ministerio de Economía y Competitividad (MINECO) (TEC201345265-R, TEC2015-71127-C2-2-R); SINFOTON-CM: S2013/MIT2790.

Acknowledgment. The work of Sonia Martin-Lopez was supported by MINECO through a "Ramón y Cajal" contract.

\section{References}

1. G. P. Agrawal, Nonlinear Fiber Optics (Academic, 2007), Ch. 9.

2. J. Urricelqui, A. Zornoza, M. Sagües, and A. Loayssa, Opt. Exp. 20, 26942 (2012).

3. A. Lopez-Gil, M. A. Soto, X. Angulo-Vinuesa, A. Dominguez-Lopez, S. Martin-Lopez, L. Thevenaz, and M. Gonzalez-Herraez, Opt. Express 24, 17200 (2016).

4. A. Lopez-Gil, X. Angulo-Vinuesa, A. Dominguez-Lopez, S. Martín-Lopez, and M. González-Herraez, Opt. Lett., 40, 2193 (2015).

5. A. Lopez-Gil, X. Angulo-Vinuesa, A. Dominguez-Lopez, S. Martin-Lopez, M. Gonzalez-Herraez, Photonics Technol. Lett. 28, 1379 (2016).

6. S. W. Lloyd, M. J. F. Digonnet, and S. Fan, J. Lightwave Technol. 31, 2070 (2013).

7. A. Zornoza, M. Sagües, and A. Loayssa, J. Lightwave Technol. 30, 1066 (2012).

8. M. Dossou, D. Bacquet, and P. Szriftgiser, Opt. Letters, 35, 3850 (2010).

9. J. Urricelqui, A. Zornoza, M. Sagües, and A. Loayssa, Opt. Exp. 22, 17403, (2014).

10. X. Lu, M. A. Soto, M. G. Herraez, and L. Thévenaz, Proc. SPIE 8794, 87943P-1, (2013).

11. G. Ryu, K. Yong Song, G. T. Kim, S. B. Lee, and K. Lee, Proc. $6^{\text {th }}$ APOS (2016).

12. A. Denisov, M. A. Soto, and L. Thévenaz, Proc. SPIE 9157, 9157D2-1 (2014).

13. A. Dominguez-Lopez, A. Lopez-Gil, S. Martin-Lopez, and M. GonzalezHerraez, IEEE Photon. Technol. Lett. 26, 338 (2014).

14. X. Angulo-Vinuesa, A. Lopez-Gil, A. Dominguez-Lopez, J.L. Cruz, M. V. Andres, S. Martin Lopez, and M. Gonzalez-Herraez, Proc. SPIE 9634, 963419-1 (2015). 


\section{Full References}

1. G. P. Agrawal, Nonlinear Fiber Optics (Academic, 2007), Ch. 9.

2. J. Urricelqui, A. Zornoza, M. Sagües, and A. Loayssa, "Dynamic BOTDA measurements based on Brillouin phase-shift and RF demodulation," Opt. Exp., vol. 20, no. 24, pp. 26942-26949, 2012.

3. A. Lopez-Gil, M. A. Soto, X. Angulo-Vinuesa, A. Dominguez-Lopez, S. Martin-Lopez, L. Thevenaz, and M. Gonzalez-Herraez, "Evaluation of the accuracy of BOTDA systems based on the phase spectral response," Opt. Express 24 (15), 17200-17214, 2016.

4. A. Lopez-Gil, X. Angulo-Vinuesa, A. Dominguez-Lopez, S. Martín-Lopez, and M. González-Herraez, "Exploiting nonreciprocity in BOTDA systems," Opt. Lett. 40 (10), 2193-2196, 2015.

5. A. Lopez-Gil, X. Angulo-Vinuesa, A. Dominguez-Lopez, S. Martin-Lopez, M. Gonzalez-Herraez, "Simple Baseband Method for the Distributed Analysis of Brillouin Phase-Shift Spectra," Photonics Technol. Lett. 28 (13), 1379-1382 (2016).

6. S. W. Lloyd, M. J. F. Digonnet, and S. Fan, "Modeling Coherent Backscattering Errors in Fiber Optic Gyroscopes for Sources of Arbitrary Line Width," J. Lightwave Technol. 31 (13), 2070-2078, 2013

7. A. Zornoza, M. Sagües, and A. Loayssa, "Self-heterodyne detection for SNR improvement and distributed phase-shift measurements in BOTDA," IEEE J. Lightwave Technol., vol. 30, no. 8, pp. 1066-1072, 2012.

8. M. Dossou, D. Bacquet, and P. Szriftgiser, "Vector Brillouin optical time-domain analyzer for high-order acoustic modes," Opt. Letters, vol. 35, no. 22, pp. 3850-3852, 2010.

9. J. Urricelqui, A. Zornoza, M. Sagües, and A. Loayssa, "Phasorial differential pulse-width pair technique for long-range Brillouin optical time-domain analysis sensors," Opt. Exp., vol. 22, no. 14, 17403-17408, 2014.

10. X. Lu, M. A. Soto, M. G. Herraez, and L. Thévenaz, "Brillouin distributed fibre sensing using phase modulated probe," Proc. SPIE, vol. 8794, pp. 87943P-1-87943P-4, 2013.

11. G. Ryu, K. Yong Song, G. T. Kim, S. B. Lee, and K. Lee, "Brillouin optical correlation domain analysis system for simultaneous interrogation of 150 sensing positions," Proc. 6th APOS, 2016.

12. A. Denisov, M. A. Soto, and L. Thévenaz, “1'000’000 resolved points along a Brillouin distributed fibre sensor," Proc. SPIE 9157, 9157D2-1 -9157D2-4, 2014.

13. A. Domınguez-Lopez, A. Lopez-Gil, S. Martın-Lopez, and M. Gonzalez-Herraez, "Signal-to-noise ratio improvement in BOTDA using balanced detection," IEEE Photon. Technol. Lett., vol. 26, no. 4, pp. 338-341, 2014.

14. X. Angulo-Vinuesa, A. Lopez-Gil, A. Dominguez-Lopez, J.L. Cruz, M. V. Andres, S. Martin Lopez, and M. Gonzalez-Herraez, "Simultaneous gain and phase profile determination on an interferometric BOTDA,"Proc. SPIE 9634, 963419-1 - 963419-4, 2015. 